\title{
Fecal Incontinence, CTCAE
}

National Cancer Institute

\section{Source}

National Cancer Institute. Fecal Incontinence, CTCAE. NCI Thesaurus. Code C143482.

A disorder characterized by inability to control the escape of stool from the rectum. 